\title{
Effects of IncRNA TUSC7 on the malignant biological behavior of osteosarcoma cells via regulation of miR-375
}

\author{
LULU WANG $^{1^{*}}$, JIANKUI JIANG $^{2 *}$, GUISEN SUN $^{1}$, PANPAN ZHANG $^{1}$ and YA LI ${ }^{1}$ \\ Departments of ${ }^{1}$ Spinal Surgery and ${ }^{2}$ Hand and Foot Surgery, \\ ShengLi Oilfield Central Hospital, Dongying, Shandong 257000, P.R. China
}

Received January 28, 2020; Accepted July 6, 2020

DOI: $10.3892 / \mathrm{ol} .2020 .11994$

\begin{abstract}
The present study aimed at investigating how long-chain non-coding RNA (lncRNA) tumor suppressor candidate 7 (TUSC7) regulates the malignant biological behavior of osteosarcoma cells. Tumor tissues and adjacent tissues of 30 patients with osteosarcoma were collected, and the expression levels of IncRNA TUSC7 and miR-375 were detected by RT-qPCR. IncRNA TUSC7 mimic and miR-375 mimic transfection models were established in MG63 osteosarcoma cells, and Transwell assays were used to detect the migration ability of MG63 cells. An MTT assay was used to assess the proliferation ability of MG63 cells. lncRNA TUSC7 in osteosarcoma tissue was significantly lower than that of adjacent tissues, while miR-375 levels were significantly higher than that of adjacent tissues; the two levels have a negative correlation. IncRNA TUSC7 mimic inhibited MG63 proliferation and migration abilities. miR-375 mimic promoted MG63 proliferation and migration abilities. The lncRNA TUSC7 mimic and miR-375 mimic co-transfection system could partially rescue the inhibition of IncRNA TUSC7 mimic on MG63 cells. In conclusion, lncRNA TUSC7 inhibited the proliferation and migration of MG63 osteosarcoma cells by regulating $\mathrm{miR}-375$.
\end{abstract}

\section{Introduction}

Osteosarcoma, a common primary malignant bone cancer, is often diagnosed in children and it is the second leading cause of cancer-related deaths of children (1). Characterized by high malignancy and recurrence rate, it affects the life of patients to a large extent. Although surgical resection and neoadjuvant

Correspondence to: Dr Guisen Sun, Department of Spinal Surgery, ShengLi Oilfield Central Hospital, 31 Jinan Road, Dongying, Shandong 257000, P.R. China

E-mail: es73ai@163.com

${ }^{*}$ Contributed equally

Key words: osteosarcoma cells, lncRNA TUSC7, miR-375, proliferation, migration chemotherapy have reduced the mortality of patients with osteosarcoma, the 5-year survival rate is still markedly low, with $10-20 \%$ of patients with metastasis $(2,3)$. Therefore, it is crucial to identify new diagnostic biomarkers and therapeutic targets, understand the influence and mechanism of these targets on the malignant biological behavior of osteosarcoma cells in order to improve the clinical treatment and the prognosis of the patients $(4,5)$.

Long-chain non-coding RNAs (lncRNAs) are RNA molecules that exist in the nucleus or cytoplasm, with a length of more than $200 \mathrm{nts}$ and have a complex secondary structure (6). lncRNAs play an important role in various biological and pathological processes, such as transcriptional regulation, cell fate determination and tumorigenesis. IncRNAs can adsorb miRNA, block miRNA, and inhibit the binding of miRNA to its target genes (6). In addition, IncRNAs plays an important role in the process of tumorigenesis and tumor progression (7). It has been revealed that numerous lncRNAs, including MEG3, HOTAIR, CCAL, tumor suppressor candidate 7 (TUSC7) play a regulatory role in the occurrence and progression of osteosarcoma at the transcriptional and post-transcriptional levels $(8,9)$, and participate in the proliferation, invasion, and migration of the tumor (10). IncRNA TUSC7 was originally identified in osteosarcoma by Pasic et al (11). The discovery of the function of lncRNA TUSC7 as a tumor-inhibiting factor in human osteosarcoma has attracted broad attention (12). Several studies have revealed that the 1ncRNA TUSC7 exhibits a downregulated expression in numerous malignant tumors, such as liver cancer (13), neuroglioma (14), colorectal cancer (15), and endometrial cancer (16). A previous study also revealed that the expression of TUSC7 in osteosarcoma was significantly lower than that in non-tumor tissues (12). lncRNA TUSC7 is a type of potential tumor-inhibiting factor, which can be used as a biomarker and the therapeutic target of osteosarcoma patients. However, how lncRNA TUSC7 regulates the proliferation of osteosarcoma cells remains unknown.

miRNAs are short non-coding RNAs ( 21 nucleotides) that can regulate gene expression at the post-transcriptional level (17). Studies have found that miR-375 can be used as a tumor therapeutic target and a tumor diagnosis mark, and it is differentially expressed in diseases such as esophageal, liver and cervical cancer, participating in the progression of the disease (17-19). A previous study revealed that miR-375 was aberrantly downregulated in osteosarcoma cells and 
functioned as a tumor suppressor (20). In addition, miR-375 has been revealed to inhibit osteogenic differentiation (21), which is involved in the growth and proliferation of osteosarcoma. A study reported that calcium-binding protein S100A6 has been revealed to promote human osteosarcoma growth by promoting cell proliferation and inhibiting osteogenic differentiation (22). The flavonoid phellodendron glycoside can inhibit the proliferation of human osteosarcoma cells and stimulate osteogenic differentiation (23). Therefore, miR-375 may promote the growth of osteosarcoma, and its role remains to be further clarified.

To the best of our knowledge, there is no previous study on the regulatory relationship between miR-375 and IncRNA TUSC7 in osteosarcoma cells. Based on the inhibitory effect of long non-coding RNA TUSC7 on the invasion of osteosarcoma cells, it was explored whether lncRNA TUSC7 can influence the malignant biological behavior of the osteosarcoma cells by regulating $\mathrm{miR}-375$.

\section{Materials and methods}

Materials. Human osteosarcoma cells (HOS and MG63) and human fetal osteoblast cells (hFOB 1.19) were purchased from ATCC. Dulbecco's modified Eagle's Medium (DMEM) and fetal bovine serum (FBS) were obtained from Gibco; Thermo Fisher Scientific, Inc. The Transwell chamber was obtained from BD Biosciences. The Two-step fluorescence quantitative PCR kit was purchased from MedChemExpress. Penicilin-streptamycin solution (double antibiotic) were obtained from Hyclone; GE Healthcare Life Sciences. Consumables such as cell culture dishes and plates were purchased from Corning Costar, Inc. TRIzol reagent was obtained from Cwbio IT Group and Lipo3000 transfection reagent was obtained from Invitrogen; Thermo Fisher Scientific, Inc..

Patient and tissue samples. The present study included 30 patients (17 males and 13 females; 15-24 years old) with osteosarcoma admitted to the Orthopedic Surgery Department of the ShengLi Oilfield Central Hospital from January 2010 to December 2019, where postoperative tissue samples were collected to analyze the pathological results. The samples were used to extract total RNA. All patients or their families signed informed and written consent. The study was approved by the ShengLi Oilfield Central Hospital Ethics Committee (approval no. SOCH20091201A).

Culture and plasmid transfection of osteosarcoma cells. HOS cells and MG63 cells were cultured in DMEM containing $10 \% \mathrm{FBS}$ and $1 \%$ penicillin/streptomycin in an $5 \% \mathrm{CO}_{2}$ and $37^{\circ} \mathrm{C}$ incubator. Although the levels of Lnc-TUSC7 in both HOS and MG63 cells were detected, the changes in MG63 cells were more significant than those of HOS cells, and subsequent experiments were performed with MG63 cells. MG63 cells were digested with $0.25 \%$ Trypsin pancreatin (Gibco; Thermo Fisher Scientific, Inc.) and $3 \times 10^{5}$ cells/well were seeded into a six-well plate. Transfection was carried out after the cell confluence reached 60-70\%. A total of $40 \mathrm{nM}$ pcDNA-TUSC7, negative control plasmid pcDNA3.1, miR-375 mimics and miR-negative control (NC) were transfected using Lipo3000 transfection reagent according to the manufacturer's instructions. After $48 \mathrm{~h}$, the total RNA of the cells was extracted to detect the transfection efficiency. Then, G418 $(500 \mu \mathrm{g} / \mathrm{ml}$, Gibco; Thermo Fisher Scientific, Inc.) was used to select cells for 4 weeks to obtain MG63 cells with stable expression of lncRNA TUSC7.

The primer sequences of the pcDNA-TUSC7 expression vector was as follows: Forward, 5'-CGATCTTAATTAAGG GGTACCAAAGTCCACTCTG-3' and reverse, 5'-TCAGTG GCGCGCCTTTTTCGTGAGTACACAATAGTCATC-3'. The miR-375 mimic sequence was as follows: Forward, 5'-CGA TCTAATTAAGGGTACCAAAGTCACTCTG-3' and reverse, 5'-TCAGGGCGCGCCTTCGTGGTACACAATAGTCAT-3'.

Transwell assay. Osteosarcoma cells with a fusion rate of $70-80 \%$ after transfection of MG63 cells in each group were collected, washed with PBS, centrifuged to remove the supernatant, and this process was repeated 2 times. Then, serum-free DMEM (Gibco; Thermo Fisher Scientific, Inc.) was added to adjust the cell concentration to $2.5 \times 10^{5}$ cells $/ \mathrm{ml}$. Subsequently, $200 \mu \mathrm{l}$ of cell suspension was added to the upper layer of a Transwell chamber prepared in advance, and the chamber was placed in a 24-well culture plate with medium containing $10 \%$ serum (Gibco; GE Healthcare Life Sciences) mixed in advance. Then, the culture plate was placed in a $37^{\circ} \mathrm{C}, 5 \% \mathrm{CO}_{2}$ incubator for $24 \mathrm{~h}$. After the culture was finished, the number of cells migrating from Transwell upper chamber to lower chamber membrane were observed under a light microscope (x20). Finally, the Transwell chamber was washed with PBS, the non-migrated cells were removed with a cotton swab and the chamber was air-dried. Then, $4 \%$ formaldehyde solution was added to the chamber for fixation at room temperature for $20 \mathrm{~min}$, and $0.1 \%$ crystal violet was added for staining at room temperature for $20 \mathrm{~min}$. Finally, the chamber was cleaned with PBS 3 times and dried naturally. The number of cells was counted and images were observed and captured under a light microscope $(x 40)$.

MTT assay. Osteosarcoma cells with a cell fusion rate of $70-80 \%$ after transfection in each group were collected and washed with PBS solution. A small number of cells was obtained and counted, and then the cell concentration was adjusted to $1 \times 10^{4}$ cells $/ \mathrm{ml}$. Diluted cell suspension (200 $\mu$ l) was seeded onto 96-well cell plates. The culture plate was placed in a $37^{\circ} \mathrm{C}, 5 \% \mathrm{CO}_{2}$ incubator for $24 \mathrm{~h}$, and then $50 \mu \mathrm{l}$ MTT solution (Beyotime Institute of Biotechnology) was added to the cell wells, cautiously avoiding light during this process. The cells were cultured for another 3-4 h, the supernatant was discarded, and $150 \mu 1$ DMSO solution was added to each well. The cells were shaken on a shaker at low speed for $10 \mathrm{~min}$ and finally the absorbance value of each well was detected at $490 \mathrm{~nm}$ with a microplate reader. Both the 48 and 72-h plates also underwent the aforementioned treatment.

Real-time qPCR assay. The transfected cells and tissues with a fusion rate of $70-80 \%$ were collected into an EP tube, and total RNA was extracted using TRIzol reagent (CoWin Biosciences). cDNA products were prepared using cDNA synthesis kit (iScript $^{\mathrm{TM}}$ cDNA kit; cat. no. 1708890; Bio-Rad Laboratories), and the reaction system was configured according to the 
specifications of the quantitative kit (SYBR Green qPCR Master Mix; cat. no. HY-K0501; MedChemExpress). The whole process $(<1 \mathrm{~h})$ was completed on ice. Then the configured reaction was placed into a tube and an ABI7500 fluorescence quantitative PCR instrument (Applied Biosystems; Thermo Fisher Scientific, Inc.) was used to detect gene expression. The PCR reaction conditions were: $95^{\circ} \mathrm{C}$ pre-denaturation for $10 \mathrm{~min} ; 95^{\circ} \mathrm{C}$ denaturation for $15 \mathrm{sec} ; 60^{\circ} \mathrm{C}$ annealing for $45 \mathrm{sec} ; 72^{\circ} \mathrm{C}$ extension for $1 \mathrm{~min} ; 40$ cycles; $72^{\circ} \mathrm{C}$ extension for $5 \mathrm{~min} ; 4^{\circ} \mathrm{C}$ retention. The relative expression of the target gene was calculated by $2^{-\Delta \Delta C q}$ method (24). The primer sequences were as follows: IncRNA TUSC7 forward, 5'-GGAAACAGA AGGCACCTCA-3' and reverse 5'-TCTCAGAGGTCAAAC AGGCA-3'; miR-375 forward, 5'-AGCCGTCAAGAGCAA TAACGAA-3' and reverse, 5'-GTGCAGGGTCCGAGGT-3'; and U6 forward, 5'-CGCTTCGGCAGCACATATAC-3' and reverse, 5'-AGGGGCCATGCTAATCTTCT-3'.

Luciferase reporter gene assay. Control and miR-375 WT, lncRNA TUSC7 and miR-375 WT, NC and miR-375 MUT, lncRNA TUSC7 and miR-375 MUT were transfected into MG63 cells (Lipo3000; Invitrogen; Thermo Fisher Scientific, Inc.), respectively, in accordance with the specifications of the luciferase reporter gene test system (Promega) kit.pmirGLO luciferase vector and luciferase reporter plasmid were constructed by GenScript. The relative fluorescence intensity of each group after $48 \mathrm{~h}$ of transfection was detected. Renilla luciferase activity was used as a standardized method.

Statistical analysis. SPSS 17.0 statistical software (SPSS, Inc.) was used to process the experimental data. An unpaired Student's t-test was used for comparison of differences between two groups, and ANOVA followed by Bonferroni test was used for comparison of differences between multiple groups. Pearson correlation analysis was also performed. The experimental results were expressed as the mean $\pm \mathrm{SD}$, and each experiment was repeated 3 times. $\mathrm{P}<0.05$ was considered to indicate a statistically significant difference; $\left({ }^{*} \mathrm{P}<0.05,{ }^{* *} \mathrm{P}<0.01,{ }^{* * *} \mathrm{P}<0.001\right.$ as indicated in the figures and legends).

\section{Results}

lncRNA TUSC7 and miR-375 are differently expressed in osteosarcoma tissue and adjacent tissue. Tissue samples and para-tumor tissues were collected from 30 patients with osteosarcoma, and qPCR was used to detect the expression level of lncRNA TUSC7 and miR-375. It was revealed that lncRNA TUSC7 in osteosarcoma tissue was significantly lower than that of para-tumor tissue $(\mathrm{P}<0.001)$, while the level of miR-375 was significantly higher than that of the adjacent tissues $(\mathrm{P}<0.001)$, and the difference was statistically significant (Fig. 1A and B). Through correlation analysis, it was revealed that there was a negative correlation between lncRNA TUSC7 and miR-375, as revealed in Fig. $1 \mathrm{C}(\mathrm{r}=-0.8119, \mathrm{P}<0.001)$. To determine the difference in the expression levels of lncRNA TUSC7, HOS, MG63 and HFOB 1.19 cell lines were selected and the expression of 1ncRNA TUSC7 was detected by RT-qPCR. The results revealed that lncRNA TUSC7 expression in HOS and MG63 cells was significantly lower than that in HFOB
1.19 cells, indicating that lncRNA TUSC7 expression was downregulated in HOS and MG63 cells (Fig. 1D).

Proliferation and migration abilities of MG63 cells transfected with lncRNA TUSC7. In order to further understand the effect of lncRNA TUSC7 on the biological function of MG63, the proliferation and migration abilities of MG63 were detected through MTT and Transwell experiments. RT-qPCR experiments revealed that in MG63 cells transfected with lncRNA pcDNA-TUSC7, the expression level of lncRNA TUSC7 was significantly enhanced compared with that in the control group, indicating that the MG63 cell line that stably expressed the target gene was successfully transfected (Fig. 2A). In addition, MTT assays and Transwell migration assays revealed that compared with the mimic control group, the proliferation (Fig. 2B) and migration (Fig. 2C and D) abilities in the 1ncRNA TUSC7 mimic group were significantly decreased. The aforementioned results indicated that lncRNA TUSC7 inhibited the proliferation and migration abilities of osteosarcoma MG63 cells to a certain extent.

Effects of miR-375 on the proliferation and migration abilities of osteosarcoma cells. After establishing the lncRNA TUSC7-mimic transfection model, the expression of miR-375 was detected and it was revealed that compared with the mimic control, miR-375 expression was significantly downregulated in the lncRNA TUSC7-mimic MG63 cells (Fig. 3A). Concurrently, it was demonstrated that miR-375 was the target gene of lncRNA TUSC7 through a luciferase reporter gene assay (Fig. 3B). In addition, MG63 cells were transfected with the miR-375 mimic and it was revealed that the expression level of miR-375 in the miR-375-mimic-transfected group was significantly higher than that of the miR-NC-transfected group (Fig. 3C). Furthermore, the proliferation capacity was significantly higher in the miR-375-mimic-transfected group than that of the miR-NC-transfected control group (Fig. 3D). The Transwell migration assay revealed that the miR-375-mimic group also had higher migratory capacity compared with the miR-NC control group (Fig. 3E and F), indicating that miR-375 promoted the growth and migration of osteosarcoma.

IncRNA TUSC7 affects the proliferation and migration of osteosarcoma cells through miR-375. miR-375 mimic and lncRNA TUSC7 mimic were co-transfected into MG63 osteosarcoma cells, and it was revealed that the proliferation ability of lncRNA TUSC7 mimic + miR-375 mimic group was significantly higher than that of lncRNA TUSC7 mimic + miR-NC group, but lower than that in the control group (mimic control+miR-NC), especially at $72 \mathrm{~h}$ (Fig. 4A). The same trend was also revealed in the Transwell migration assay. The migration ability of the lncRNA TUSC7 mimic + miR-375 mimic group was significantly higher than that of the lncRNA TUSC7 mimic + miR-NC group (Fig. 4B and C), indicating that miR-375 mimic can partially rescue the inhibitory effect of lncRNA TUSC7 on osteosarcoma.

\section{Discussion}

Osteosarcoma (OS) is a common primary bone neoplasm and one of the most invasive solid malignant tumors (1). Evidently, 

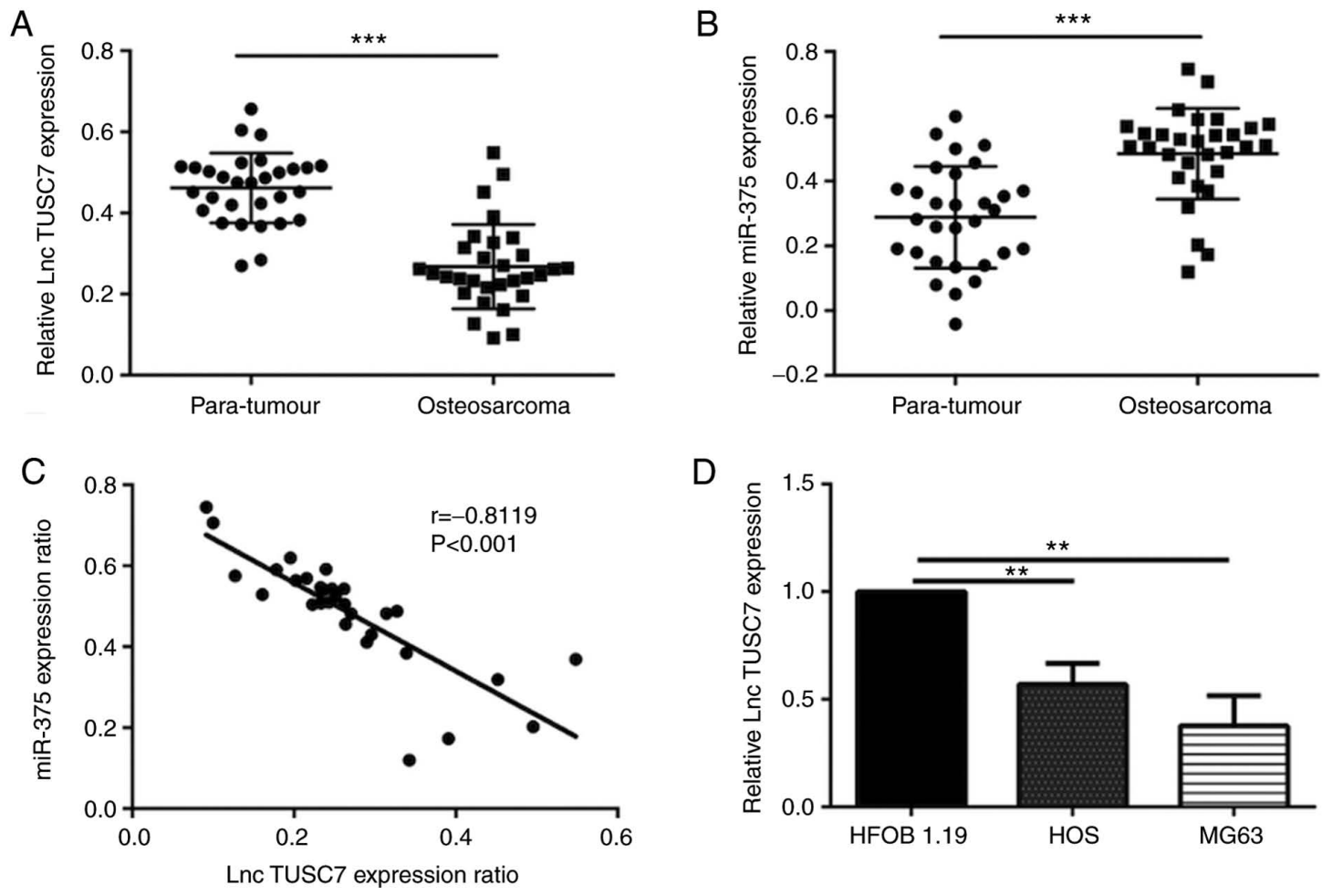

Figure 1. lncRNA TUSC7 and miR-375 are differently expressed in osteosarcoma tissue and adjacent tissue. (A) The expression level and comparison of lncRNA TUSC7 in osteosarcoma tissue and adjacent tissue. (B) The expression level and comparison of miR-375 in osteosarcoma tissue and adjacent tissue. (C) Correlation analysis of lncRNA TUSC7 and miR-375 expression levels in osteosarcoma tissues of patients. (D) Comparison of the expression difference of lncRNA TUSC7 between HFOB 1.19, HOS and MG63 cells. ${ }^{* *} \mathrm{P}<0.01$ and ${ }^{* * *} \mathrm{P}<0.001$. IncRNA, long-chain non-coding RNA; TUSC7, tumor suppressor candidate 7.
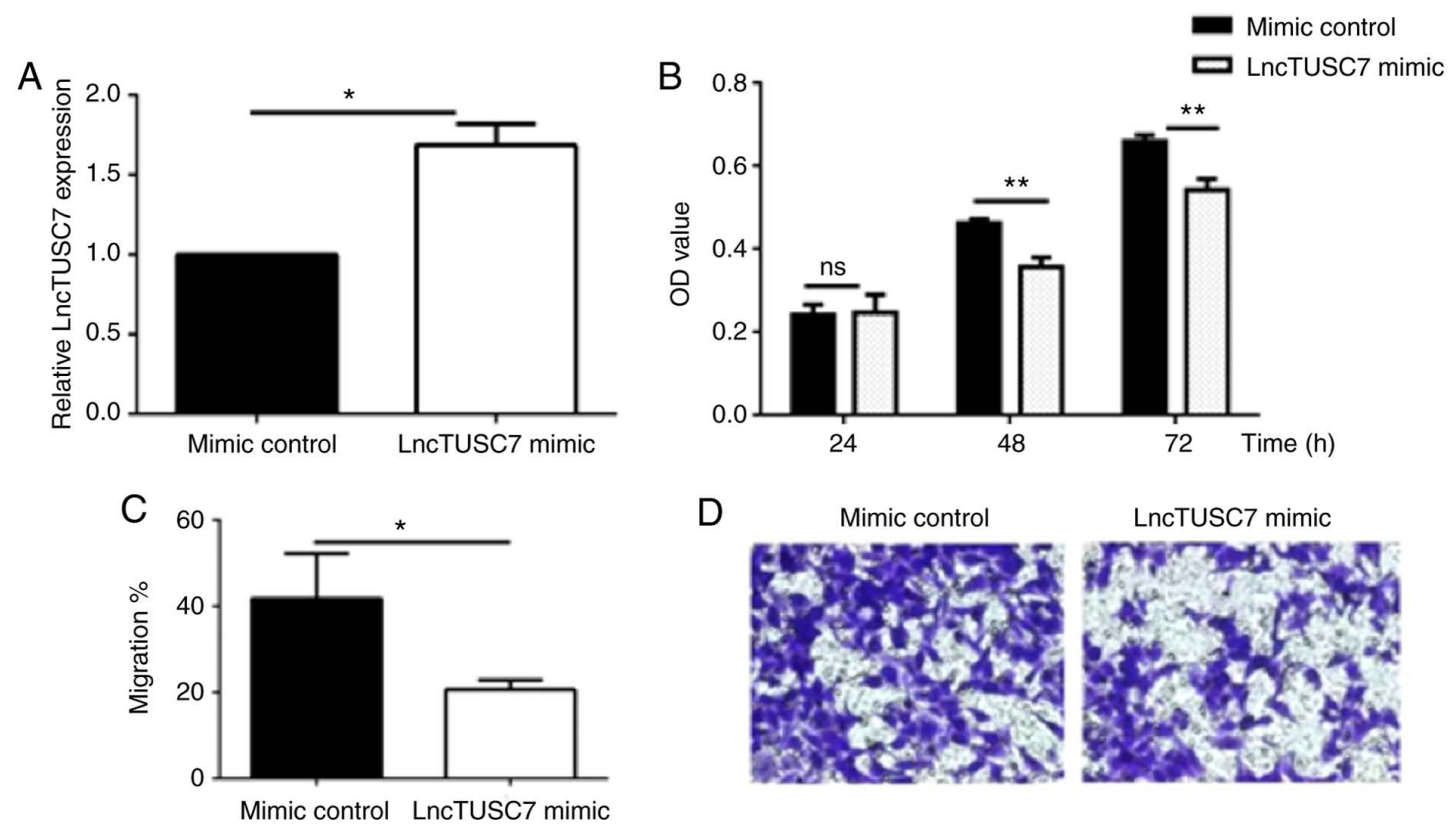

$\mathrm{D}$

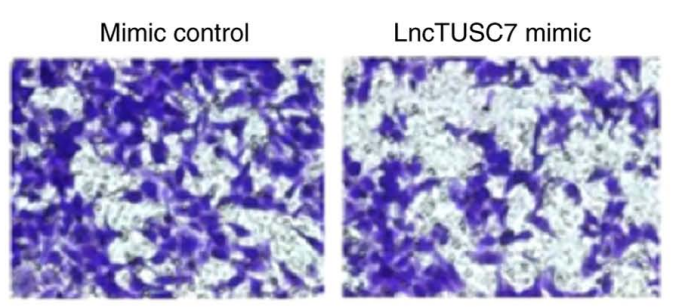

Figure 2. Effects of lncRNA TUSC7 on the proliferation and migration of MG63 and validation of transfection efficiency. (A) After the MG63 cells were transfected with lncRNA TUSC7 mimic, the transfection efficiency was detected through RT-qPCR. (B) After the MG63 cells were transfected with lncRNA TUSC7 mimic, the proliferation ability of MG63 cells was detected through MTT assay. (C and D) After the MG63 cells were transfected with lncRNA TUSC7 mimic, the migration ability of MG63 cells was detected through Transwell assay. ${ }^{*} \mathrm{P}<0.05$ and ${ }^{* *} \mathrm{P}<0.01$. lncRNA, long-chain non-coding RNA; TUSC7, tumor suppressor candidate 7.

the occurrence of osteosarcoma is correlated with numerous factors such as race, age, sex, various genomic changes, and the environment people live in (25). However, to date, the cause of the disease is still controversial (1-4). In the present study, it was determined that lncRNA TUSC7 in osteosarcoma tissue was significantly lower than that in the adjacent tissue, while 

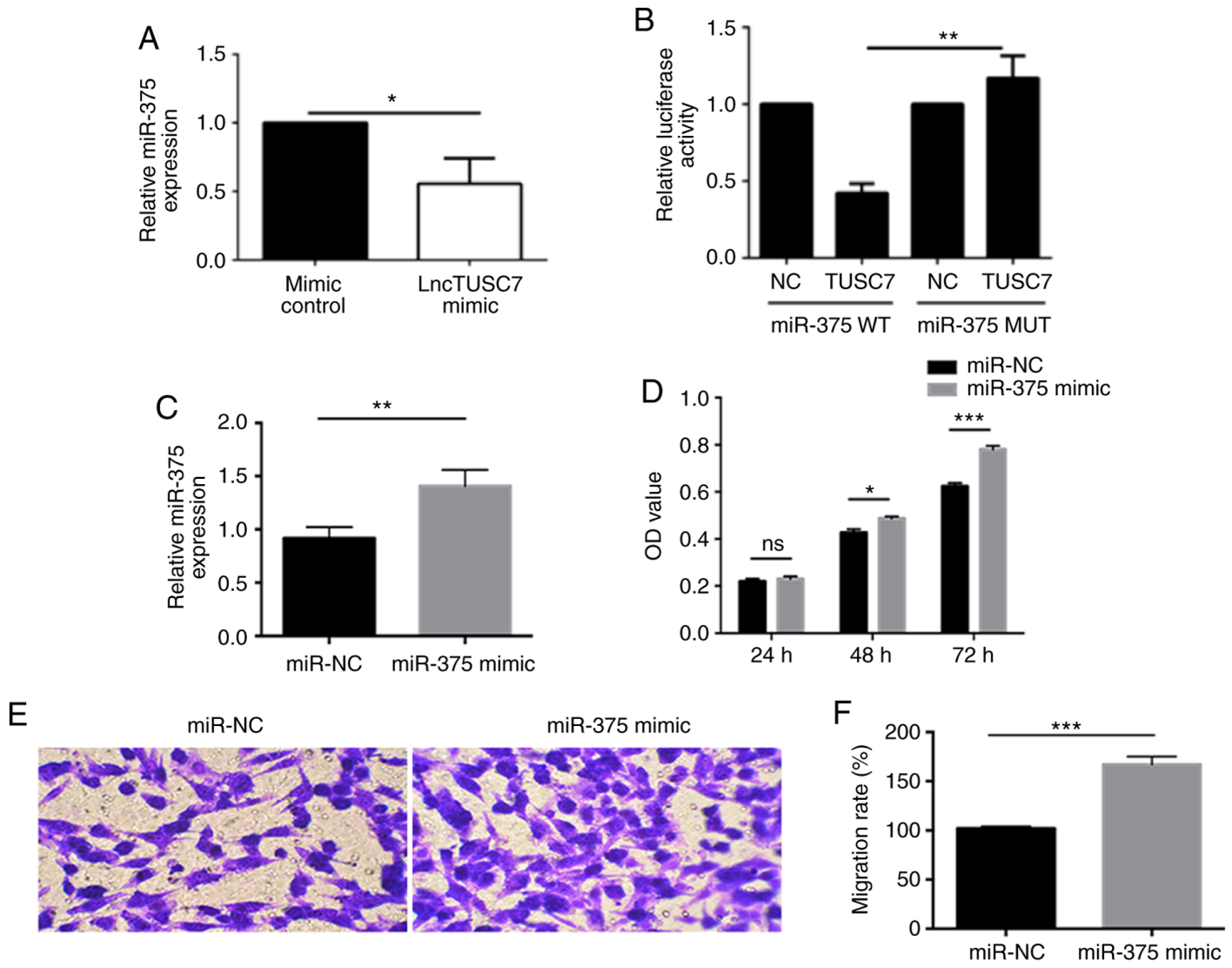

Figure 3. Effects of overexpression of 1ncRNA TUSC7 on miR-375 and on cell proliferation and migration of MG63 cells. (A) After transfected with 1ncRNA TUSC7 mimic, the expression of miR-375 was detected through qPCR. (B) The relationship between miR-375 and lncRNA TUSC7 was verified through the luciferase reporter gene assay. (C) The expression level of miR-375 in MG63 cells after transfection with miR-375 mimic. (D) After transfection with miR-375 mimic, an MTT assay was used to detect the proliferation ability of MG63 cells. (E and F) After transfection with miR-375 mimic, a Transwell migration assay was used to detect the migration ability of MG63 cells. ${ }^{*} \mathrm{P}<0.05,{ }^{* *} \mathrm{P}<0.01$ and ${ }^{* * * *} \mathrm{P}<0.001$. lncRNA, long-chain non-coding RNA; TUSC7, tumor suppressor candidate 7.
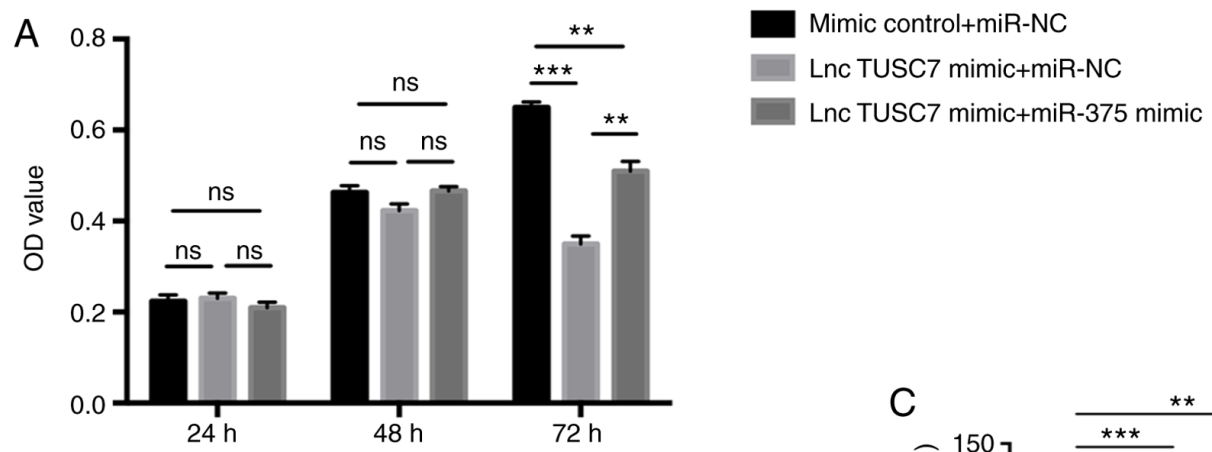

$\mathrm{B}$
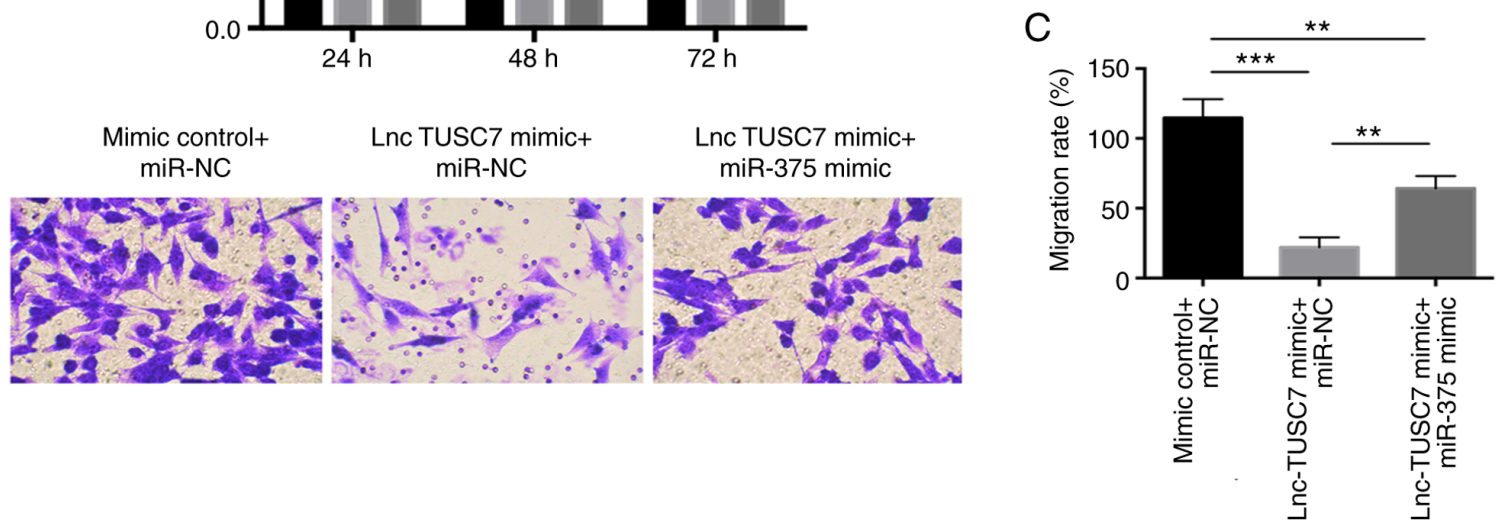

Figure 4. Changes in the proliferation and migration abilities of MG63 after transfection with 1ncRNA TUSC7 mimic and miR-375 mimic. (A) An MTT experiment was used to detect the proliferation ability of MG63 after concurrent transfection with lncRNA TUSC7 mimic and miR-375 mimic. (B and C) A Transwell migration assay was used to detect the change of the migration ability after concurrent transfection with lncRNA TUSC7 mimic and miR-375 mimic. ${ }^{* *} \mathrm{P}<0.01$ and ${ }^{* * * *} \mathrm{P}<0.001$. IncRNA, long-chain non-coding RNA; TUSC7, tumor suppressor candidate 7 . 
miR-375 level in osteosarcoma tissue was significantly higher than that in the adjacent tissue, and there was a negative correlation between the two. The expression differences of two types of osteosarcoma cell lines (HOS and MG63) and the normal osteoblast HFOB 1.19 cell line were examined. It was revealed that the expression of IncRNA TUSC7 in osteosarcoma cells was lower than that in normal cells, especially in MG63 cells. The present study confirmed that IncRNA TUSC7 exhibited an antitumor effect in osteosarcoma cell line MG63. IncRNAs are a type of RNA, defined as regulatory transcripts that have no-protein coding potential and are more than 200 nucleotides in length $(16,26,27)$. By regulating genetic expression at the transcriptional, post-transcriptional or epigenetic levels, it has been revealed that lncRNAs participate in cell activities and the formation of various diseases (28). According to the ENCODE project (29), more than 90 percent of the human genome can be transcribed, and only about two percent of the genome are protein-coding genes (30). Therefore, it has been revealed that most transcriptomes are of noncoding transcripts, including microRNAs, IncRNAs, and circular RNAs (30). As oncogenes or cancer suppressor genes, IncRNAs play an important role in the process of tumor development $(30,31)$.

TUSC7 consists of four exons that are located on chromosome $3 q 13.3$ (11). Studies have demonstrated that TUSC7 exhibits a downregulated expression in most cancers and plays an anticancer role (15). TUSC7 has been revealed to inhibit the process of the epithelial-mesenchymal transition (13) in liver cancer by negatively regulating miR-10a. The overexpression of TUSC7 inhibited the occurrence of glioma and gastric cancer by targeting miR-23b (14). The upregulation of TUSC7 has been revealed to inhibit the proliferation of lung cancer, colorectal cancer and osteosarcoma (15). These aforementioned studies revealed that TUSC7 can play an anticancer role by sponging certain tumor-related miRNAs. In the present study, a transfection cell model with lncRNA TUSC7 mimic was set up to detect the influence of lncRNA TUSC7 on the malignant biological behavior of MG63 cells. We successfully constructed the MG63 cell line expressing lncRNA TUSC7, and also used qPCR experiments to verify that the expression level of lncRNA TUSC7 in the lncRNA TUSC7-mimic group was significantly upregulated. Then, the effect of lncRNA TUSC7 on the migration ability of the MG63 osteosarcoma cells was assessed using Transwell migration assays. The results revealed that in comparison with the control group, the migration ability of MG63 cells transfected with the lncRNA TUSC7 mimic was significantly downregulated. Similarly, the MTT assay revealed that the proliferation ability of the lncRNA TUSC7-mimic group was significantly lower than that of the mimic control group, indicating that lncRNA TUSC7 could inhibit the growth of MG63 cells. Collectively the aforementioned results indicated that TUSC7 can function as a type of cancer suppressor gene in osteosarcoma cells. TUSC7 with high expression can inhibit the proliferation rate and the migration rate of tumor cells.

It has been demonstrated that the aberrant expression of miR-375 is correlated with the occurrence and progression of a variety of cancers, including colorectal (15), lung (14), oral $(18,20)$ and breast cancer $(19)$, as well as glioma (32) and gastric cancer (33). It has been revealed that the expression of miR-375 is increased in gastric cancer, yet miR-375 can desensitize cells to ionizing radiation and etoposide via targeting of p53 (33). miR-375 has been revealed to downregulate human colorectal cancer cell lines and tissues, and inhibit the growth of colorectal cancer cells (34-36). In addition, it has been revealed that miR-375 inhibits osteogenic differentiation (21), and studies have confirmed that tumor growth can be inhibited by promoting osteogenic differentiation $(22,23)$. Therefore, miR-375 may promote the growth of osteosarcoma. The present study revealed that miR-375 expression in tumor tissue of osteosarcoma patients was reduced, and miR-375 mimic could enhance the malignant biological behavior of MG63 cells. It was revealed that miR-375 plays a role in promoting the growth of osteosarcoma, which is consistent with the results of some previous studies $(22,23)$.

lncRNAs are reported to regulate gene expression, chromosome modification, or the function of miRNAs (37) through binding to RNA-binding proteins. In the present study it was further confirmed through a luciferase reporter gene assay that miR-375 is the target gene of lncRNA TUSC7, and that co-transfection of lncRNA TUSC7 mimic and miR-375 mimic could partially rescue the inhibitory effect of lncRNA TUSC7 on MG63 cells, confirming that lncRNA TUSC7 inhibits the malignant biological behavior of osteosarcoma by regulating the expression of miR-375. The specific mechanism of how lncRNA TUSC7 and miR-375 affects the biological behavior of osteosarcoma needs to be further clarified. It is suggested that miR-375 inhibits osteogenic differentiation and lncRNA TUSC7 may promote osteogenic differentiation by inhibiting the expression of miR-375. In addition, miR-375 has a variety of target genes, which involve complex cell signaling pathway regulation processes, and requires further exploration. In the future, extensive data using bioinformatics will be used to confirm the binding site between 1ncRNA TUSC7 and miR-375, and the mechanism will be further studied through the method of binding site mutation. 1ncRNA TUSC7 may inhibit osteosarcoma cell growth by acting on miR-375, which provides us with a new perspective for treating patients.

\section{Acknowledgements}

Not applicable.

Funding

No funding was received.

\section{Availability of data and materials}

The datasets used and/or analyzed during the present study are available from the corresponding author on reasonable request.

\section{Authors' contributions}

LW, JJ and GS conceived and designed the study, and drafted the manuscript. LW, JJ, PZ and YL collected, analyzed and interpreted the experimental data. GS revised the manuscript for important intellectual content. All authors read and approved the final manuscript. 


\section{Ethics approval and consent to participate}

The study was approved by the Ethics Committee of ShengLi Oilfield Central Hospital (approval no. SOCH20091201A).

\section{Patient consent for publication}

Not applicable.

\section{Competing interests}

The authors declare that they have no competing interests.

\section{References}

1. Lin YH, Jewell BE, Gingold J, Lu L, Zhao R, Wang LL and Lee DF: Osteosarcoma: Molecular pathogenesis and iPSC modeling. Trends Mol Med 23: 737-755, 2017.

2. Wang J, Liu S, Shi J, Li J, Wang S, Liu H, Zhao S, Duan K, Pan X and Yi Z: The role of miRNA in the diagnosis, prognosis, and treatment of osteosarcoma. Cancer Biother Radiopharm 34: 605-613, 2019.

3. Wan J, Zhang X, Liu T and Zhang X: Strategies and developments of immunotherapies in osteosarcoma. Oncol Lett 11 : 511-520, 2016

4. Lindsey BA, Markel JE and Kleinerman ES: Osteosarcoma overview. Rheumatol Ther 4: 25-43, 2017.

5. Geller DS and Gorlick R: Osteosarcoma: A review of diagnosis, management, and treatment strategies. Clin Adv Hematol Oncol 8: 705-718, 2010

6. Xu S, Gong Y, Yin Y, Xing $\mathrm{H}$ and Zhang N: The multiple function of long noncoding RNAs in osteosarcoma progression, drug resistance and prognosis. Biomed Pharmacother 127: 110141, 2020.

7. LiZ, Shen J, Chan MT and Wu WK: TUG1: A pivotal oncogenic long non-coding RNA of human cancers. Cell Prolif 49: 471-475, 2016.

8. Li N, Shi K and Li W: TUSC7: A novel tumor suppressor long non-coding RNA in human cancers. J Cell Physiol 233 6401-6407, 2018.

9. Hung T and Chang HY: Long noncoding RNA in genome regulation: Prospects and mechanisms. RNA Biol 7: 582-585, 2010

10. Wang B, Su Y, Yang Q, Lv D, Zhang W, Tang K, Wang H, Zhang R and Liu Y: Overexpression of long non-coding RNA HOTAIR promotes tumor growth and metastasis in human osteosarcoma. Mol Cells 38: 432-440, 2015.

11. Pasic I, Shlien A, Durbin AD, Stavropoulos DJ, Baskin B, Ray PN Novokmet A and Malkin D: Recurrent focal copy-number changes and loss of heterozygosity implicate two noncoding RNAs and one tumor suppressor gene at chromosome 3q13.31 in osteosarcoma. Cancer Res 70: 160-171, 2010.

12. Cong M, Li J, Jing R and Li Z: Long non-coding RNA tumor suppressor candidate 7 functions as a tumor suppressor and inhibits proliferation in osteosarcoma. Tumour Biol 37 : 9441-9450, 2016.

13. Wang Y, Liu Z, Yao B, Dou C, Xu M, Xue Y, Ding L, Jia Y, Zhang $\mathrm{H}, \mathrm{Li} \mathrm{Q}$, et al: Long non-coding RNA TUSC7 acts a molecular sponge for miR-10a and suppresses EMT in hepatocellular carcinoma. Tumour Biol 37: 11429-11441, 2016.

14. Shang C, Guo Y, Hong Y and Xue YX: Long non-coding RNA TUSC7, a target of miR-23b, plays tumor-suppressing roles in human gliomas. Front Cell Neurosci 10: 235, 2016.

15. Ren W, Chen S, Liu G, Wang X, Ye H and Xi Y: TUSC7 acts as a tumor suppressor in colorectal cancer. Am J Transl Res 9 . 4026-4035, 2017.

16. Wu X, Cai D, Zhang F, Li M and Wan Q: Long noncoding RNA TUSC7 inhibits cell proliferation, migration and invasion by regulating SOCS4 (SOCS5) expression through targeting miR-616 in endometrial carcinoma. Life Sci 231: 116549, 2019.

17. Krol J, Loedige I and Filipowicz W: The widespread regulation of microRNA biogenesis, function and decay. Nat Rev Genet 11: 597-610, 2010.

18. Siow MY, Ng LP, Vincent-Chong VK, Jamaludin M, Abraham MT, Abdul Rahman ZA, Kallarakkal TG, Yang YH, Cheong SC and Zain RB: Dysregulation of miR-31 and miR-375 expression is associated with clinical outcomes in oral carcinoma. Oral Dis 20: 345-351, 2014.
19. Luo D, Wilson JM, Harvel N, Liu J, Pei L, Huang S, Hawthorn L and Shi H: A systematic evaluation of miRNA:mRNA interactions involved in the migration and invasion of breast cancer cells. J Transl Med 11: 57, 2013.

20. Wu X, Ajani JA, Gu J, Chang DW, Tan W, Hildebrandt MA, Huang M, Wang KK and Hawk E: MicroRNA expression signatures during malignant progression from Barrett's esophagus to esophageal adenocarcinoma. Cancer Prev Res (Phila) 6: 196-205, 2013.

21. Du F, Wu H, Zhou Z and Liu YU: microRNA-375 inhibits osteogenic differentiation by targeting runt-related transcription factor 2. Exp Ther Med 10: 207-212, 2015.

22. Li Y, Wagner ER, Yan Z, Wang Z, Luther G, Jiang W, Ye J, Wei Q, Wang J, Zhao L, et al: The calcium-binding protein S100A6 accelerates human osteosarcoma growth by promoting cell proliferation and inhibiting osteogenic differentiation. Cell Physiol Biochem 37: 2375-2392, 2015.

23. Zhang N, Ying MD, Wu YP, Zhou ZH, Ye ZM, Li H and Lin DS Hyperoside, a flavonoid compound, inhibits proliferation and stimulates osteogenic differentiation of human osteosarcoma cells. PLoS One 9: e98973, 2014.

24. Livak KJ and Schmittgen TD: Analysis of relative gene expression data using real-time quantitative PCR and the 2(-Delta Delta C(T)) method. Methods 25: 402-408, 2001

25. Prater S and Mckeon B: Cancer, osteosarcoma. In: StatPearls [Internet]. Treasure Island (FL): StatPearls Publishing, 2020. https://www.ncbi.nlm.nih.gov/pubmed/31751058.

26. Liu Y, Xing R, Zhang X, Dong W, Zhang J, Yan Z, Li W, Cui J and $\mathrm{Lu} \mathrm{Y}$ : miR-375 targets the p53 gene to regulate cellular response to ionizing radiation and etoposide in gastric cancer cells. DNA Repair (Amst) 12: 741-750, 2013.

27. Chang Y, Yan W, He X, Zhang L, Li C, Huang H, Nace G, Geller DA, Lin J and Tsung A: miR-375 inhibits autophagy and reduces viability of hepatocellular carcinoma cells under hypoxic conditions. Gastroenterology 143: 177-187 e8, 2012.

28. Song W, Xie J, Li J, Bao C and Xiao Y: The emerging roles of long noncoding RNAs in bone homeostasis and their potential application in bone-related diseases. DNA Cell Biol 39: 926-937, 2020.

29. ENCODE ProjectConsortium, Birney E,Stamatoyannopoulos JA, Dutta A, Guigó R, Gingeras TR, Margulies EH, Weng Z, Snyder M, Dermitzakis ET, et al: Identification and analysis of functional elements in $1 \%$ of the human genome by the ENCODE pilot project. Nature 447: 799-816, 2007.

30. Esteller M: Non-coding RNAs in human disease. Nat Rev Genet 12: 861-874, 2011.

31. Ling H, Vincent K, Pichler M, Fodde R, Berindan-Neagoe I, Slack FJ and Calin GA: Junk DNA and the long non-coding RNA twist in cancer genetics. Oncogene 34: 5003-5011, 2015.

32. Chang C, Shi H, Wang C, Wang J, Geng N, Jiang X and Wang X: Correlation of microRNA-375 downregulation with unfavorable clinical outcome of patients with glioma. Neurosci Lett 531: 204-208, 2012

33. Xu Y, Deng Y, Yan X and Zhou T: Targeting miR-375 in gastric cancer. Expert Opin Ther Targets 15: 961-972, 2011.

34. Elshafei A, Shaker O, Abd El-Motaal O and Salman T: The expression profiling of serum miR-92a, miR-375, and miR-760 in colorectal cancer: An Egyptian study. Tumour Biol 39: $1010428317705765,2017$.

35. Cui F, Wang S, Lao I, Zhou C, Kong H, Bayaxi N, Li J, Chen Q, Zhu T and Zhu H: miR-375 inhibits the invasion and metastasis of colorectal cancer via targeting SP1 and regulating EMT-associated genes. Oncol Rep 36: 487-493, 2016.

36. Xu L, Li M, Wang M, Yan D, Feng G and An G: The expression of microRNA-375 in plasma and tissue is matched in human colorectal cancer. BMC Cancer 14: 714, 2014.

37. Arab K, Park YJ, Lindroth AM, Schäfer A, Oakes C, Weichenhan D, Lukanova A, Lundin E, Risch A, Meister M, et al: Long noncoding RNA TARID directs demethylation and activation of the tumor suppressor TCF21 via GADD45A. Mol Cell 55: 604-614, 2014.

This work is licensed under a Creative Commons Attribution-NonCommercial-NoDerivatives 4.0 International (CC BY-NC-ND 4.0) License. 